\title{
High-resolution structure determination of the CylR2 homodimer using paramagnetic relaxation enhancement and structure-based prediction of molecular alignment
}

\author{
Sigrun Rumpel $\cdot$ Stefan Becker $\cdot$ Markus Zweckstetter
}

Received: 24 April 2007/Accepted: 14 September 2007/Published online: 20 November 2007

(C) Springer Science+Business Media B.V. 2007

\begin{abstract}
Structure determination of homooligomeric proteins by NMR spectroscopy is difficult due to the lack of chemical shift perturbation data, which is very effective in restricting the binding interface in heterooligomeric systems, and the difficulty of obtaining a sufficient number of intermonomer distance restraints. Here we solved the high-resolution solution structure of the $15.4 \mathrm{kDa}$ homodimer CylR2, the regulator of cytolysin production from Enterococcus faecalis, which deviates by $1.1 \AA$ from the previously determined X-ray structure. We studied the influence of different experimental information such as long-range distances derived from paramagnetic relaxation enhancement, residual dipolar couplings, symmetry restraints and intermonomer Nuclear Overhauser Effect restraints on the accuracy of the derived structure. In addition, we show that it is useful to combine experimental information with methods of ab initio docking when the available experimental data are not sufficient to obtain convergence to the correct homodimeric structure. In particular, intermonomer distances may not be required when residual dipolar couplings are compared to values predicted on the basis of the charge distribution and the shape of ab initio docking solutions.
\end{abstract}

Electronic supplementary material The online version of this article (doi:10.1007/s10858-007-9204-4) contains supplementary material, which is available to authorized users.

S. Rumpel · S. Becker · M. Zweckstetter $(\square)$ Department for NMR-based Structural Biology, Max Planck Institute for Biophysical Chemistry, Am Fassberg 11, 37077 Gottingen, Germany

e-mail: mzwecks@gwdg.de
Keywords NMR solution structure - Homodimer CylR2 . Paramagnetic relaxation enhancement - PALES . Residual dipolar couplings · Rigid-body docking

\section{Introduction}

Oligomerization plays an important role in protein function and many soluble and membrane proteins form homodimers or higher oligomers (Goodsell and Olson 2000). The structure of oligomeric proteins in solution can be determined by NMR spectroscopy, which is particularly important for lowaffinity complexes that are difficult to crystallize (Vaynberg and Qin 2006). In principle, the same NMR approach can be followed for complexes as for monomeric proteins, i.e. analysis of a 3D heteronuclear-edited NOESY (Nuclear Overhauser Enhancement Spectroscopy) on the basis of a nearly complete resonance assignment. However, the difficulty to distinguish intramonomer from intermonomer correlations, the increasing size of protein-protein complexes and the requirement to determine structures in a costand time-efficient manner motivated the development of various rigid-body docking approaches such as HADDOCK (Dominguez et al. 2003). Rigid-body docking requires knowledge of the 3D structure of the individual molecules and can be driven by a small number of NOEs, residual dipolar couplings (RDCs), ambiguous intermolecular distance restraints from chemical shift perturbation, long-range distances derived from saturation transfer or paramagnetic probes and other biological data (Clore 2000; Diaz-Moreno et al. 2005; Dominguez et al. 2003; Matsuda et al. 2004).

For homooligomeric systems, structure determination is complicated in three ways. Firstly, preparation of samples formed by a defined mixture of protein with different isotope labels often requires unfolding and refolding of the 
homooligomer. Secondly, intermolecular NOEs are especially difficult to observe due to the inherent symmetry of the system. Thirdly, as the protein cannot be obtained easily in a monomeric form, it is generally not possible to map the binding interface using chemical shift perturbation data, hydrogen exchange dynamics or backbone and side chain dynamics (Englander et al. 1997; Kay et al. 1996). To overcome this problem, paramagnetic relaxation agents might be added to the solution (Petros et al. 1990; Sakakura et al. 2005) or saturation transfer experiments in mixtures of unlabeled and ${ }^{15} \mathrm{~N} /{ }^{2} \mathrm{H}$-labeled protein may be recorded (Takahashi et al. 2000). Very often, however, the information obtained from these experiments is ambiguous or the experiments fail due to insufficient sensitivity (Liepinsh et al. 2001).

Accurate intermolecular distance and orientational restraints between atom pairs are important for the determination of high-resolution structures of protein-protein complexes. Gaponenko et al. added sub-stoichiometrically a paramagnetic probe to break the symmetry and observe monomer specific pseudocontact shifts and RDCs in a homodimer (Gaponenko et al. 2002). A drawback of the approach is that the number of signals and signal overlap is strongly increased due to the broken symmetry and pseudocontact shifts. Alternatively, long-range structural information may be derived from paramagnetic relaxation enhancement (PRE) observed in the presence of a paramagnetic nitroxide radical that has been specifically attached to a diamagnetic protein (Kosen 1989). PRE derived distances are highly useful for structural characterization of globular (Bertini et al. 1996a, b, 1997; Donaldson et al. 2001; Feeney et al. 2001; Gaponenko et al. 2000) and intrinsically disordered proteins (Dyson and Wright 1998), as well as protein-protein (Iwahara and Clore 2006) and protein-DNA complexes (Iwahara et al. 2004).

When the available experimental data are not sufficient to obtain convergence to the correct complex structure, experimental data might be combined with algorithms designed for ab initio protein-protein docking (Russell et al. 2004). For example, chemical shift perturbation data alone (Morelli et al. 2001) or in combination with RDCs (Dobrodumov and Gronenborn 2003) were applied to filter the correct structure from ab initio docking results. In homooligomeric systems, however, chemical shift perturbation data and monomer specific RDCs are not easily accessible.

RDCs, however, offer an alternative way of rapidly validating structures or models of proteins and proteinprotein complexes. RDCs can be observed in proteins that are weakly aligned in an anisotropic environment (Tjandra and Bax 1997). The preferred orientation of the protein and the observed RDC values depend on the three-dimensional shape and electrostatic properties of the biomolecule. Based on this insight, we had developed a simple simulation method, called PALES, that allows prediction of a protein's alignment tensor with reasonable accuracy from the three-dimensional charge distribution and shape of the macromolecule (Zweckstetter and Bax 2000; Zweckstetter et al. 2004). Recently, we showed that PALES in combination with RDCs that were observed in a charged Pf1 alignment medium can be used to rapidly determine the relative orientation and stoichiometry of coiled-coil proteins in solution. In particular, antiparallel homodimers could be unambiguously distinguished from parallel coiled-coil homodimers (Zweckstetter et al. 2005).

Enterococcus faecalis is one of the major causes for hospital-acquired antibiotic-resistant infections. The $15.4 \mathrm{kDa}$ homodimer CylR2 is part of a two-component system that regulates the production of the exotoxin cytolysin (Gilmore et al. 1990; Murray 1990; Haas et al. 2002). We previously reported the X-ray structure of CylR2, showed its role as a repressor of cytolysin transcription and proposed a model of the CylR2/DNA complex structure (Rumpel et al. 2004). Here we (i) determined the highresolution structure of CylR2 in solution, (ii) studied the influence of different experimental intermonomer information on the accuracy of the derived structure and (iii) showed that despite the lack of sufficient experimental data the 3D structure of CylR2 can be determined by including information from ab initio docking and prediction of molecular alignment as implemented in PALES.

\section{Materials and methods}

\section{NMR sample preparation}

Details of cloning, protein overexpression and purification have been described elsewhere (Razeto et al. 2004). Single cysteine mutants of CylR2 (N40C and T55C) were generated by using the QuikChange site-directed mutagenesis kit (Stratagene). The introduced mutations were confirmed by DNA sequencing. ${ }^{15} \mathrm{~N}$ - and ${ }^{13} \mathrm{C} /{ }^{15} \mathrm{~N}$-labeled samples were prepared from Escherichia coli cells grown in M9-based minimal medium containing ${ }^{15} \mathrm{NH}_{4} \mathrm{Cl}$ and/or ${ }^{13} \mathrm{C}_{6}$-glucose. Samples to determine intermonomer distances were prepared by dissolving ${ }^{15} \mathrm{~N}$-labeled $w t$ and unlabeled mutant in $8 \mathrm{M}$ urea, mixing them in a 1:1 molar ratio and refolding by dialysis against $50 \mathrm{mM}$ HEPES $\mathrm{pH}$ 7.0, $600 \mathrm{mM} \mathrm{NaCl}$ and $5 \mathrm{mM}$ DTT. Directly before labeling with MTSL ((1-oxy-2,2,5,5-tetramethyl-D-pyrroline-3-methyl)-methanethiosulfonate, Toronto Research Chemicals), DTT was removed by using size exclusion chromatography (PD-10 columns, Amersham Pharmacia Biosciences). Free sulfhydryl groups were modified overnight at room temperature with a 3-5 fold molar excess MTSL, solubilized in acetone. Unreacted MTSL 
was removed via a PD-10 column. Complete incorporation of MTSL was confirmed by mass spectrometry. Following NMR analysis in the oxidized form, samples were reduced by adding a 2-3 molar excess of $200 \mathrm{mM}$ ascorbic acid.

\section{NMR spectroscopy}

NMR samples contained $0.4-0.8 \mathrm{mM}$ CylR2 in $50 \mathrm{mM}$ HEPES pH 7.0, $600 \mathrm{mM} \mathrm{NaCl}$ and 5\% $\mathrm{D}_{2} \mathrm{O}(\mathrm{v} / \mathrm{v})$. All NMR experiments were acquired at $298 \mathrm{~K}$ on Bruker AVANCE 600 or 700 or DRX 600 spectrometers. NMR experiments used for resonance assignment, for measurement of residual dipolar couplings and for calculation of ${ }^{15} \mathrm{~N}-{ }^{1} \mathrm{H}-\mathrm{NOE}$ values were performed as described (Rumpel et al. 2004). For structure determination a $3 \mathrm{D}\left[{ }^{15} \mathrm{~N},{ }^{1} \mathrm{H}\right] \mathrm{NOESY}-\mathrm{HSQC}$ and a $\left[{ }^{13} \mathrm{C},{ }^{1} \mathrm{H}\right] \mathrm{NOESY}-\mathrm{HSQC}$ with a mixing time of $120 \mathrm{~ms}$ were measured. 2D $\left[{ }^{15} \mathrm{~N},{ }^{1} \mathrm{H}\right] \mathrm{HSQC}$ and ${ }^{15} \mathrm{~N} T_{2}$ relaxation experiments were performed for site-directed spin-labeling studies. The $T_{2}$ relaxation times were sampled using seven different ${ }^{15} \mathrm{~N}$ relaxation delays: 7.6, 50, 90, 130, 160, 190 and $220 \mathrm{~ms}$. Rotating frame relaxation times $\left(\mathrm{T}_{1 \rho}\right)$ of backbone nitrogens were estimated from two 1D spectra with a relaxation delay of 2 and $60 \mathrm{~ms}$ and with a spin-lock power of $2.5 \mathrm{kHz}$. All spectra were processed using NMRPipe/NMRDraw (Delaglio et al. 1995) and analyzed using Sparky (T. D. Goddard and D. G. Kneller, University of California, San Francisco).

Structure calculation of the CylR2 monomer

The previously reported resonance assignment (Rumpel et al. 2004) and torsion angle restraints as predicted from chemical shifts with the software TALOS (Cornilescu et al. 1999) were used as input for combined automated NOE assignment and structure calculation with the program CYANA (Guntert 2004). For the final CYANA run 19 ${ }^{13} \mathrm{C}$-distances (three short range, nine medium range and seven long range) were assigned manually. The final 20 structures with the lowest target function were used for further refinement in the presence of HN-RDCs and in explicit solvent using Xplor-NIH (Schwieters et al. 2003).

Long-range distances from PRE broadening

For each of the two single-cysteine containing mutants, CylR $2_{\mathrm{N} 40 \mathrm{C}}$ and CylR2 $2_{\mathrm{T} 55 \mathrm{C}}$, two samples were used (molecules carrying spin labels are indicated by a star), a pure ${ }^{15} \mathrm{~N}$ - and spin-labeled homodimer $\left({ }^{15} \mathrm{~N}-\operatorname{mut}(*) /{ }^{15} \mathrm{~N}-\mathrm{mut}(*)\right)$ and a 1:1 mixture of ${ }^{15} \mathrm{~N}$-labeled $w t$ and spin-labeled mutant at natural abundance $\left(1: 1\right.$-mixed $\left.{ }^{15} \mathrm{~N}-w t / \operatorname{mut}(*)\right)$.
Intra and intermolecular PRE distances were obtained from intensities of cross-peaks of backbone amide protonnitrogen pairs in ${ }^{15} \mathrm{~N}-\mathrm{HSQC}$ spectra of the paramagnetic $\left(I_{\text {para }}\right)$ and diamagnetic $\left(I_{\text {dia }}\right)$ state (i.e. after addition of ascorbic acid). Intensity ratios $I_{\text {para }} / I_{\text {dia }}$ were linearly fit for the enhancement of the transverse relaxation rate by the unpaired electron $\left(R_{2}^{\text {para }}\right)$ (Battiste and Wagner 2000):

$\frac{I_{\text {para }}}{I_{\text {dia }}}=\frac{R_{2} \exp \left(-R_{2}^{\text {para }} t\right)}{R_{2}+R_{2}^{\text {para }}}$,

in which $t$ is the total INEPT evolution time of the ${ }^{15} \mathrm{~N}$ HSQC $(\sim 11.3 \mathrm{~ms})$ and amide proton $R_{2}$ values were approximated by experimental amide nitrogen $R_{2}$ values (Ishima and Torchia 2003). The distances $r$ between the unpaired electron and the amide protons was determined according to

$r=\left[\frac{K}{R_{2}^{\text {para }}}\left(4 \tau_{c}+\frac{3 \tau_{c}}{1+\omega_{h}^{2} \tau_{c}^{2}}\right)\right]^{1 / 6}$,

in which $K$ is $1.23 \times 10^{-32} \mathrm{~cm}^{6} \mathrm{~s}^{-2}$ and $\omega_{h}$ is the Larmor frequency of the proton. $\tau_{c}$ is the correlation time for the electron-nuclear interaction that was assumed to be equal to the global correlation time of CylR2, which was estimated as $6 \mathrm{~ns}$ using Stokes' law (Cavanagh 1996). Changing $\tau_{c}$ from 6 to $4 \mathrm{~ns}$ did not change the docking results significantly, in agreement with the small (compared to $r$ ) influence of $\tau_{c}$ on the calculated distance (Eq. 2).

The 1:1-mixed ${ }^{15} \mathrm{~N}-w t / m u t(*)$ samples are composed of three different dimers: $50 \%{ }^{15} \mathrm{~N}-w t / m u t(*)$ heterodimer, $25 \%{ }^{15} \mathrm{~N}-\left.w t\right|^{15} \mathrm{~N}-w t$ homodimer and $25 \% \operatorname{mut}(*) / \operatorname{mut}(*)$ homodimer (Fig. 1a). When the chemical shifts of an amide-amide proton pair in the ${ }^{15} \mathrm{~N}-w t /$ mut $(*)$ heterodimer are identical to the values in the ${ }^{15} \mathrm{~N}-w t{ }^{15} \mathrm{~N}-w t$ homodimer, the ${ }^{15} \mathrm{~N}-\left.w t\right|^{15} \mathrm{~N}-w t$ homodimer contributes $50 \%$ of the NMR signal intensity even in the paramagnetic state of the 1:1-mixed ${ }^{15} \mathrm{~N}-w t /$ mut $\left({ }^{*}\right)$ sample. This was taken into account by calculation of $I_{\text {para }}$ according to

$I_{\text {para }}=2\left(I_{\text {para* }}-\frac{I_{\mathrm{dia}}}{2}\right)$,

in which $I_{\mathrm{para}} *$ is the signal intensity in the spectrum of the paramagnetic state.

Determination of the structure of the CylR2 homodimer

To allow usage of PRE-derived intermolecular distances in rigid-body docking, we explicitly included MTSL in the atomic coordinates of the monomeric structure of CylR2. MTSL molecules were attached simultaneously to $\mathrm{N} 40 \mathrm{C}$ and T55C. Starting from the structure of CylR2 $2_{\mathrm{N} 40 \mathrm{C}}+\mathrm{T} 55 \mathrm{C}$, we repeated the structure calculation 


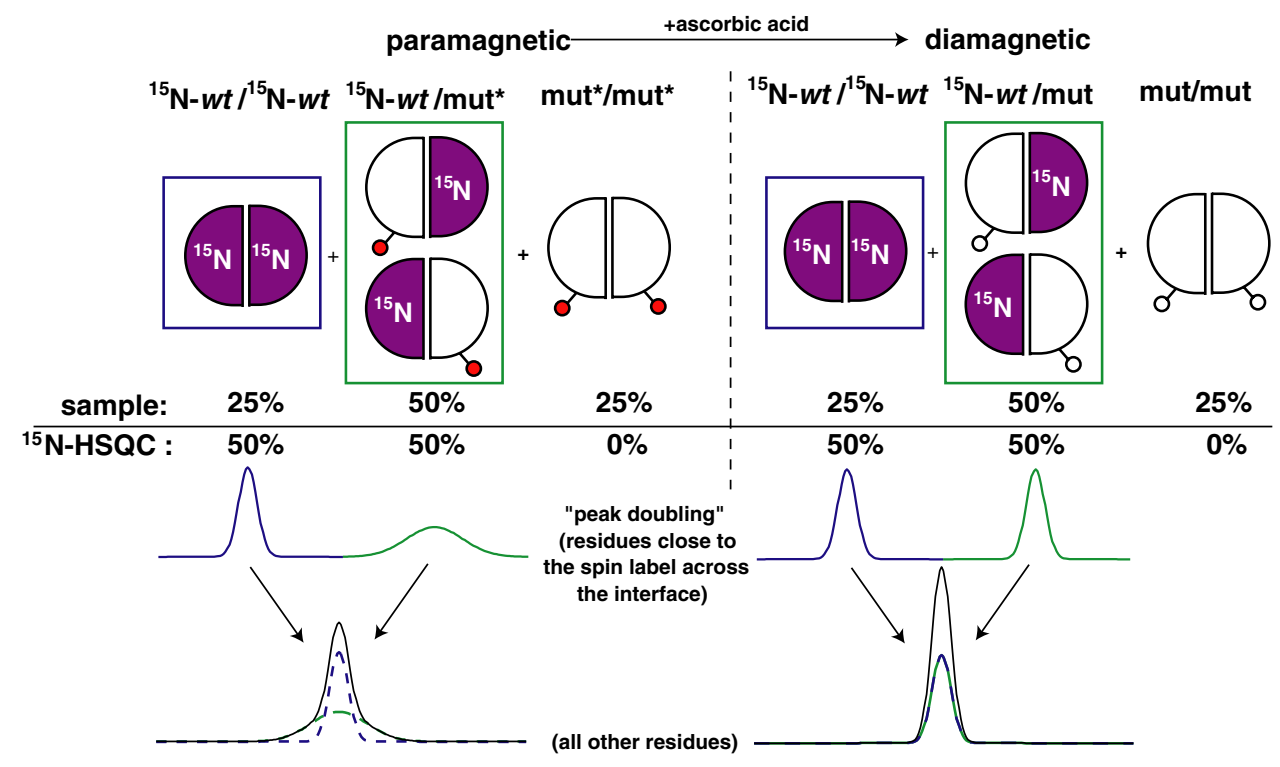

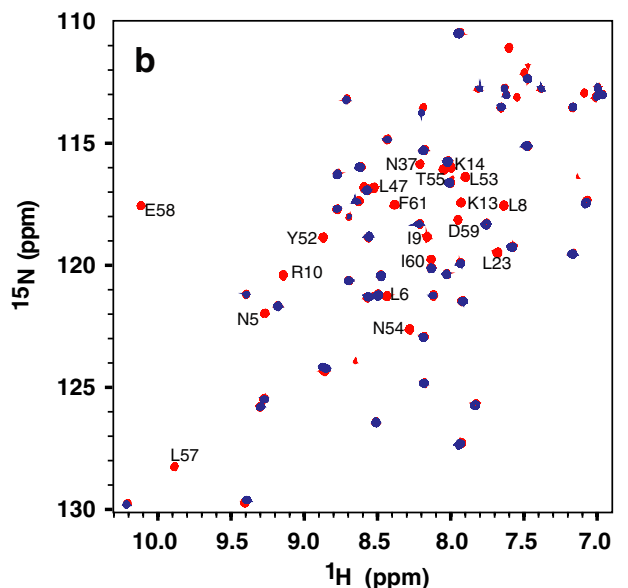

Fig. 1 (a) Overall strategy to derive intermonomer distances from PRE in homodimers. The paramagnetic sample is shown on the left side with a star to indicate paramagnetic subunits and the diamagnetic sample is shown on the right side. The 1:1-mixed samples are composed of equal amounts of ${ }^{15} \mathrm{~N}$-labeled $w t\left({ }^{15} \mathrm{~N}-w t\right.$, violet $)$ and of paramagnetic mutant $(\operatorname{mut}(*)$, white) monomers. The monomers combine into three distinct dimerization pairs: $25 \%{ }^{15} \mathrm{~N}-w t /{ }^{15} \mathrm{~N}-w t$ (blue), $50 \%{ }^{15} \mathrm{~N}-w t / \operatorname{mut}(*)$ (green) and $25 \% \operatorname{mut}(*) / \operatorname{mut}(*)$. The former two species contribute equally to the NMR signal while the latter is undetected. For a few residues close to the para or diamagnetic tag across the dimer interface, the chemical shift can

of the monomer of CylR2 using CYANA. In addition to the restraints, which were already used for calculation of the structure of $w t$ CylR2, we included intramolecular PRE distances between the spin label and the amide protons. Intramolecular PRE distances were derived from PRE broadening effects observed in the ${ }^{15} \mathrm{~N}-\operatorname{mut}(*) /{ }^{15} \mathrm{~N}-\operatorname{mut}(*)$ homodimer sample. To avoid inclusion of intermolecular effects, we analyzed the signals of only those residues

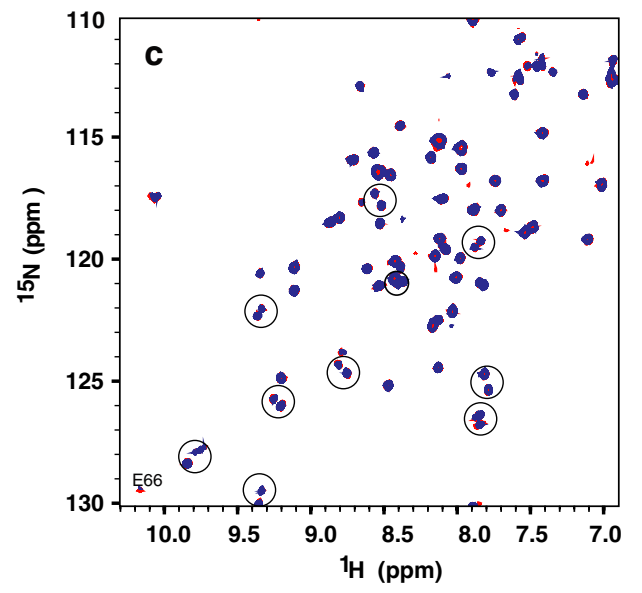

be distinguished (peak-doubling), while for all other residues, the ${ }^{15} \mathrm{~N}-w t /{ }^{15} \mathrm{~N}-w t$ and ${ }^{15} \mathrm{~N}-w t /$ mut $(*)$ peaks overlap. The PRE distance is derived from the peak intensity ratio $\left(\mathrm{I}_{\text {para }} / \mathrm{I}_{\mathrm{dia}}\right)$ obtained from the paramagnetic and diamagnetic lines (green lines). For the overlapped case, $\mathrm{I}_{\text {para }}$ can be obtained by subtracting $\mathrm{I}_{\text {dia }} / 2$ according to Eq. 3 . The diamagnetic sample can easily be obtained from the paramagnetic sample by ascorbic acid reduction. (b, c) Overlay of ${ }^{15} \mathrm{~N}-{ }^{1} \mathrm{H}$ HSQC spectra of paramagnetic (blue) and diamagnetic (red) forms of ${ }^{15} \mathrm{~N}$-mut CylR $2_{\mathrm{T} 55 \mathrm{C}}(\mathbf{b})$ and the $1: 1$ mixed ${ }^{15} \mathrm{~N}-w t /$ mut CylR $2_{\mathrm{T} 55 \mathrm{C}}(\mathbf{c})$. Residues that disappeared in the paramagnetic state are labeled and doubled peaks in (c) are indicated by ellipses

that did not show broadening in the ${ }^{15} \mathrm{~N}-w t / m u t(*)$ sample, resulting in 24 and 31 PRE restraints for CylR2 ${ }_{\mathrm{N} 40 \mathrm{C}}$ and $\mathrm{CylR} 2_{\mathrm{T} 55 \mathrm{C}}$, respectively. PRE restraints were enforced as upper limit restraints that were obtained by addition of $5 \AA$ to the distances calculated according to Eq. 2. For peaks broadened beyond detection, the upper distance limit was set to $12 \AA$ in CYANA calculations. 
Homodimer structures were calculated using a protocol for rigid-body docking as implemented in Xplor-NIH (Clore 2000). Four different sets of restraints were used: (a) PRE, (b) PRE and RDCs, (c) PRE, NOE and RDCs, and (d) NOE and RDCs. Additional calculations were performed enforcing twofold symmetry using distance difference restraints (Nilges 1993). Thirteen peaks of the ${ }^{13} \mathrm{C}-\mathrm{NOESY}$ HSQC were manually assigned as intermonomer NOEs. For NOE data, upper and lower distances were set to +2.5 and $-2 \AA$ of the calculated distances, respectively. With the exception of (d) all calculations were performed using two monomers that had MTSL attached at N40C and T55C (see above). Intermonomer PRE distances were restrained from the nitrogen of the MTSL ring in one monomer to the amide protons of the other monomer. Upper and lower distance bounds were set to $\pm 5 \AA$ of the distances calculated according to Eq. 2. Decreasing the error bounds to $\pm 4 \AA$ resulted in an increased rmsd and in a larger number of violated intermolecular restraints. For peaks broadened beyond detection, distances were set to $7 \pm 5 \AA$ A. For residues with broadened signals that are in the primary sequence next to a residue, which was not affected by PRE, only a lower distance bound was enforced. For residues that were not broadened in the paramagnetic state, a lower distance bound of $25 \AA$ was used.

The structures obtained from rigid-body docking were further refined in explicit water using Xplor-NIH (Schwieters et al. 2003). For this aim the MTSL-containing monomers in the homodimer structure were replaced by the atomic coordinates of the $w t$ protein. To restrain the monomer-to-monomer orientation, all intermolecular $\mathrm{HN}-\mathrm{HN}$ distances from $\mathrm{N} 40$ and $\mathrm{T} 55$ to any amide proton of the other subunit were extracted and restrained during refinement (error bounds of $\pm 2 \AA$ ). In addition, the intramonomer distance restraints, which had been used for calculation of the monomer structure of the $w t$ protein, were included into the refinement. Coordinates of backbone atoms and atoms of side chains not contributing to the dimer interface (as determined on the basis of the homodimeric structure prior to refinement) were fixed during refinement. The ensemble of 15 lowest energy structures, which was calculated on the basis of intermolecular PRE distances and RDCs, was deposited in the ProteinDataBank database (PDB accession code: 2GZU).

$\mathrm{Ab}$ initio docking in case of insufficient experimental restraints

$\mathrm{Ab}$ initio docking (i.e. without experimental restraints) of two monomeric CylR2 molecules was performed for both the monomeric mean structure of the NMR ensemble and a monomer of the X-ray structure using the DOT algorithm
(Mandell et al. 2001) available on the ClusPro Web server (http://www.nrc.bu.edu/cluster). The symmetry was restricted to $\mathrm{C}_{2}$ (Comeau and Camacho 2005). The docking solutions produced by DOT were ranked using ClusPro, which uses electrostatic and desolvation energies (Comeau et al. 2004).

For each docking model, the distances $\left(d^{\text {dock }}\right)$ between the $\mathrm{C} \beta$ atom of the cysteine, to which the MTSL was attached, and the backbone amide protons of the other monomer were calculated using MOLMOL (Koradi et al. 1996). $d^{\text {dock }}$ distances were compared to experimental distances $\left(d^{\text {exp }}\right)$ obtained from PRE broadening in MTSLtagged, paramagnetic CylR $2_{\mathrm{N} 40 \mathrm{C}}$ and $\mathrm{CylR} 2_{\mathrm{T} 55 \mathrm{C}}$ according to

$\sigma=\frac{1}{N} \sum_{i=1}^{N} \sqrt{\left(d_{i}^{\text {dock }}-d_{i}^{\text {exp }}\right)^{2}}$,

in which $\mathrm{N}$ is the number of residues.

RDCs were predicted using the electrostatic alignment method as implemented in the software PALES (Zweckstetter et al. 2004). The default charge was attached to all ionizable residues, the Pf1 concentration was set to $12 \mathrm{mg} \mathrm{ml}^{-1}$ and the ionic strength was adjusted to $0.5 \mathrm{M}$ $\mathrm{NaCl}$. The agreement of 35 experimental RDCs (located in secondary structure elements) with ab initio docking models was evaluated using Pearson's linear correlation coefficient.

\section{Results and discussion}

Structure of the monomeric subunit of CylR2 in solution

The structure of the monomeric subunit of the 66-residue protein CylR2 was solved based on a $98.8 \%$ complete chemical shift assignment, 987 interproton distances, 86 dihedral angle restraints and $57 \mathrm{HN}$-RDCs (Table 1). There were no major differences between the X-ray and the NMR structure (Supplementary text and Fig. S1).

Mutagenesis and spin-labeling of CylR2

To enable measurement of long-range distances in CylR2, single cysteine residues were introduced into wt CylR2. Conservative sites of mutation were chosen at position N40 and T55. N40 and T55 were located in loop regions and on opposite sides of the structure of the monomer (as was known from the NMR structure of the monomeric subunit of CylR2). As the dimer interface is not known initially, mutations might be located in the dimer interface and 
Table 1 Structural statistical data for the monomeric subunit of CylR2 ${ }^{\mathrm{a}}$

\begin{tabular}{|c|c|}
\hline \multicolumn{2}{|l|}{ NOE distance restraints } \\
\hline Total & 987 \\
\hline Short range $(|i-j| \leq 1)$ & 594 \\
\hline Medium range $(1<|i-j|<5)$ & 180 \\
\hline Long range $(|i-j| \leq 5)$ & 213 \\
\hline Dihedral angle restraints & 86 \\
\hline${ }^{15} \mathrm{~N}-{ }^{1} \mathrm{H}$ residual dipolar couplings & 57 \\
\hline \multicolumn{2}{|l|}{ Mean rmsd from experimental restraints ${ }^{b}$} \\
\hline NOE & $0.0048 \AA$ \\
\hline Dihedral angles & $0.743^{\circ}$ \\
\hline \multicolumn{2}{|l|}{ Average number of ${ }^{b}$} \\
\hline NOE violations $>0.5 \AA$ & 0 \\
\hline Dihedral angle violations $>5^{\circ}$ & 0 \\
\hline \multicolumn{2}{|c|}{ Mean rmsd from idealized covalent geometry ${ }^{b}$} \\
\hline Bonds & $0.0097 \AA$ \\
\hline Angles & $1.35^{\circ}$ \\
\hline Impropers & $1.62^{\circ}$ \\
\hline \multicolumn{2}{|l|}{ Ramachandran analysis $^{c}$} \\
\hline Most-favored region & $88.5 \%$ \\
\hline Additionally allowed region & $10.3 \%$ \\
\hline Generously allowed region & $1.0 \%$ \\
\hline Disfavored region & $0.3 \%$ \\
\hline \multicolumn{2}{|l|}{ Rmsd from the mean (residues $3-63)^{\mathrm{d}}$} \\
\hline Backbone atoms & $0.60 \pm 0.14 \AA$ \\
\hline All heavy atoms & $1.06 \pm 0.12 \AA$ \\
\hline \multicolumn{2}{|c|}{ Rmsd from the X-ray structure (residues $3-63)^{\mathrm{d}}$} \\
\hline Backbone atoms & $0.89 \AA$ \\
\hline All heavy atoms & $1.88 \AA$ \\
\hline \multicolumn{2}{|c|}{ a Statistics are for the final 20 simulated annealing structures } \\
\hline \multicolumn{2}{|c|}{ b Evaluated with Xplor-NIH (Schwieters et al. 2003) } \\
\hline \multicolumn{2}{|c|}{ c Calculated with PROCHECK-NMR (Laskowski et al. 1996) } \\
\hline${ }^{\mathrm{d}}$ Determined with MOLMOL (Korac & \\
\hline
\end{tabular}

destabilize the oligomeric structure. Therefore, we compared chemical shifts and ${ }^{15} \mathrm{~N}$ transverse relaxation times between $w t$ and mutant CylR2. With the exception of L57 in case of $\mathrm{CylR} 2_{\mathrm{N} 40 \mathrm{C}}$ and residues around the mutation site, averaged amide proton and amide nitrogen chemical shift differences between $w t$ and mutant CylR2 were smaller than $0.16 \mathrm{ppm}$. $T_{1 \rho}$ values indicated an unchanged state of oligomerization (data not shown). Thus, introduction of a cysteine at N40 and T55 did not strongly perturb the structure of CylR2.

Overnight incubation of CylR $2_{\mathrm{N} 40 \mathrm{C}}$ and $\mathrm{CylR} 2_{\mathrm{T} 55 \mathrm{C}}$ with MTSL resulted in efficient attachment of the spin label to the protein. In both the ${ }^{15} \mathrm{~N}$-mut $(*) /{ }^{15} \mathrm{~N}$-mut(*) homodimer and ${ }^{15} \mathrm{~N}-w t /$ mut $(*)$ sample signal intensities of several residues were attenuated in the paramagnetic state. Overall, intermonomer PRE-broadening as measured in the 1:1-mixed ${ }^{15} \mathrm{~N}-w t / \operatorname{mut}(*)$ sample was less pronounced. Only the signal of E66 was no longer observed, while one third of all backbone amide signals disappeared in case of ${ }^{15} \mathrm{~N}$-mut $(*) /{ }^{15} \mathrm{~N}$-mut(*) due to strong intramolecular PRE (Fig. 1b). In the 1:1-mixed ${ }^{15} \mathrm{~N}$-wt/mut(*) heterodimer, peak-doubling was observed for 10 of the 62 backbone amide signals, indicative of differences in the chemical environment close to the mutation site (Fig. 1c).

\section{Long-range distances from PRE}

Distance information derived from PREs has three advantages over NOEs: (i) It is long-range and not limited to the dimer interface, (ii) it can be used in the case of fully deuterated proteins or for proteins for which no side chain assignment can be obtained and, (iii) the number of accessible distances might be increased by attaching spin labels to different sites in the protein (at the expense of an increased amount of biochemical work). Intermonomer distances in CylR2 were derived from peak intensities of HSQC spectra recorded for the paramagnetic and diamagnetic 1:1-mixed ${ }^{15} \mathrm{~N}$-wt/mut(*) sample. Two cases had to be distinguished. For residues affected by doubling of peaks, the peak corresponding to the heterodimeric ${ }^{15} \mathrm{~N}-w t /$ mut(*) mutant was identified as the signal that was shifted compared to the ${ }^{15} \mathrm{~N}$-HSQC of $w t$ CylR2 and this peak was used for calculation of the intermonomer distance according to Eq. 2. For residues without doubling of the peak, $50 \%$ of the intensity of the peak in the diamagnetic ${ }^{15} \mathrm{~N}-\mathrm{HSQC}$, corresponding to the contribution of the ${ }^{15} \mathrm{~N}-w t /{ }^{15} \mathrm{~N}-w t$ homodimer, was subtracted from the intensity of the same peak observed in the ${ }^{15} \mathrm{~N}$-HSQC of the paramagnetic sample (Eq. 3). This approach is valid under the assumption that the sample contains $50 \%{ }^{15} \mathrm{~N}-w t /$ mut $(*)$ and $25 \%{ }^{15} \mathrm{~N}-w t /{ }^{15} \mathrm{~N}-w t$, i.e. both contribute $50 \%$ of the signal intensity (Fig. 1a).

To assess the accuracy of experimentally determined intermonomer PRE distances, we initially compared them to distances present in the X-ray structure of CylR2 (Fig. 2a). Overall there is good agreement and most experimental PRE distances deviate by less than $5 \AA$ from the values observed in the X-ray structure. The remaining deviations can have a variety of sources. (i) The structure of CylR2 in solution deviates slightly from the structure in the crystalline state. (ii) Amide proton $T_{2}$ relaxation times were approximated by experimental amide nitrogen $T_{2}$ relaxation times (Ishima and Torchia 2005). (iii) The correlation time for the electron-nuclear interaction $\tau_{c}$ was assumed to be equal to the global correlation time of CylR2. (iv) Positional averaging of the flexible nitroxide side chain of MTSL (please see "Discussion" below). (v) Errors in the determination of protein concentration and 
interference of MTSL with dimer formation during refolding from $8 \mathrm{M}$ urea may result in a deviation from the $50 \%$ contribution of ${ }^{15} \mathrm{~N}-w t /$ mut $(*)$ and ${ }^{15} \mathrm{~N}-w t /{ }^{15} \mathrm{~N}-w t$ to the ${ }^{15} \mathrm{~N}-\mathrm{HSQC}$ signal, which was assumed in the determination of intermonomer distances for residues that showed a single peak in the ${ }^{15} \mathrm{~N}-w t / \operatorname{mut}(*)$ sample. Note that errors in peak intensities have a more pronounced influence on calculated distances when the intensity reduction due to the paramagnetic center is small (Fig. 2b).

High-resolution structure of the CylR2 homodimer in solution

The structure of the CylR2 homodimer in solution was determined by rigid-body docking of two copies of the high-resolution NMR structure of the monomeric subunit

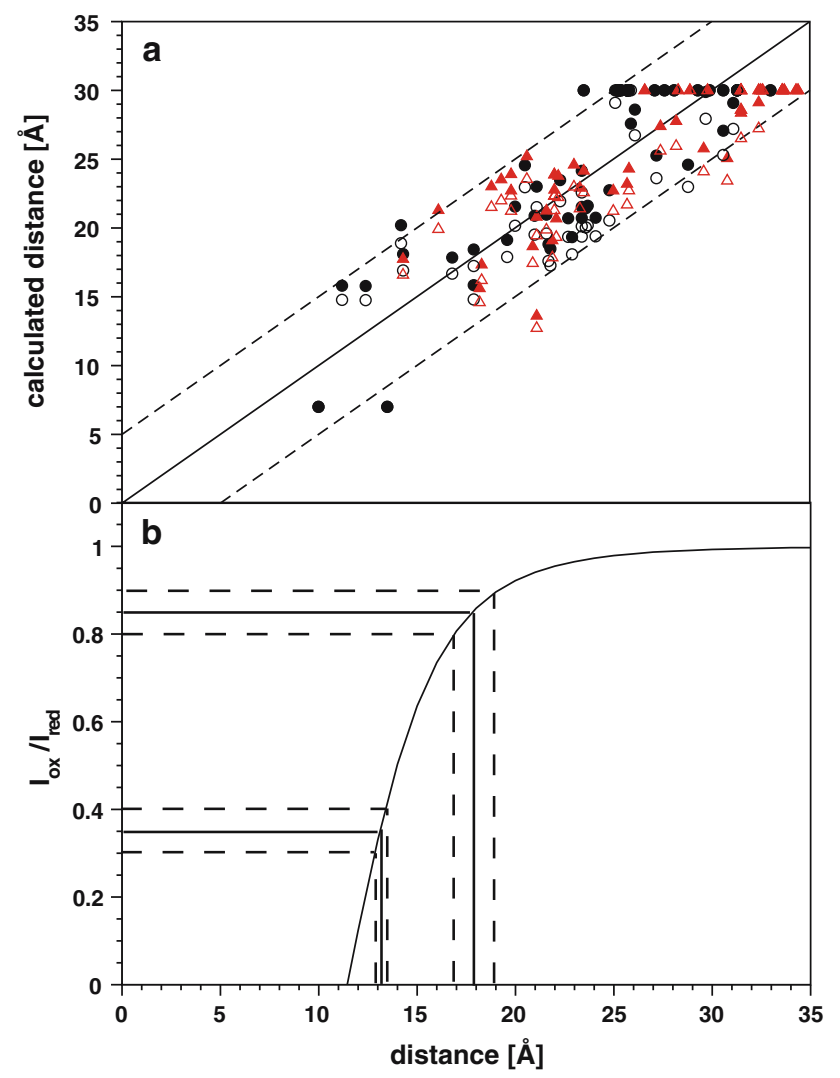

Fig. 2 (a) Theoretically expected distances from the X-ray structure versus distances calculated from PRE data. The solid line indicates optimal correlation between experimental and expected distances and the dashed line marks the $\pm 5 \AA$ error bounds. Distances calculated with the spin-label at position $\mathrm{N} 40 \mathrm{C}$ and $\mathrm{T} 55 \mathrm{C}$ are shown as black circles and red triangles, respectively. Distances calculated with a $\tau_{c}$ of 6 and 4 ns are indicated as filled and empty symbols, respectively. (b) Measured intensity ratio plotted as a function of the calculated distance. The dashed lines show that for an intensity ratio of $0.85 \pm 0.05$ the uncertainty of the distance is approximately four times larger than for an intensity ratio of $0.35 \pm 0.05$ of CylR2. Rigid-body docking of heterodimeric proteinprotein (Gray 2006) and protein-DNA complexes (van Dijk et al. 2006) is well established. In particular, the HADDOCK protocol is highly popular and was employed for several applications, in which various types of intermonomer restraints were used (see for example Dominguez et al. 2004; Volkov et al. 2005). We followed a protocol similar to HADDOCK implemented in Xplor$\mathrm{NIH}$ (Clore 2000) to obtain answers to four questions: (i) How do different types of intermonomer restraints influence the accuracy of the structure of CylR2 obtained by rigid-body docking? (ii) What is the high-resolution structure of CylR2 in solution and does it differ from the previously determined X-ray structure? (iii) Is it possible to use PRE distances obtained from only one cysteine mutant or can intermolecular distance information be removed completely and near-native solutions identified using molecular alignment prediction?

Rigid-body docking of CylR2 monomers was performed using (a) PREs, (b) PREs and RDCs, (c) PREs, NOE and RDCs, and (d) NOEs and RDCs. The backbone of the structure that was calculated using only PREs deviated by $3.0 \AA$ from the X-ray structure (Table 2). Enforcing twofold symmetry using distance difference restraints (Nilges 1993) did not change the accuracy of the structure, as PREs were already defined as symmetric restraints between the two subunits of CylR2 during rigid-body docking (data not shown). Inclusion of HN-RDCs reduced the deviation from the crystal structure to $1.5 \AA$. This is in agreement with the fact that in the presence of RDCs, one of the principal axes of the alignment tensor must be parallel and the other two orthogonal to the twofold symmetry axis (Bewley and Clore 2000). Combination of PREs and HN-RDCs with 13 intermolecular NOEs slightly further reduced the deviation from the X-ray structure. On the other hand, when only 13 intermolecular NOEs and 57 backbone HN-RDCs were used, the rigid-body docking solutions deviated by about $2 \AA$ from the X-ray structure (Table 2). The results demonstrate the power of combining long-range distance information with RDC-derived orientational information for structure determination of homooligomeric proteins.

Structures obtained from rigid-body docking were further refined in explicit water (see "Materials and methods" for details). This resulted in ensembles of 20 lowest energy structures with coordinate precision in the range from 0.54 to $0.59 \AA$ (Fig. 3a). The coordinates of the backbone and side chain atoms of the mean structure deviated by 1.15 and $2.08 \AA$ from the values in the X-ray structure. The rmsd values between the NMR and the X-ray structure were slightly higher for the dimer than for the monomer, indicative of small differences in the orientations of the two monomers within the two structures (Tables 1,2). Most notable are the differences for the longest helix $\alpha 4$ 
Table 2 Influence of different types of intermonomer restraints on the accuracy of the homodimeric structure of CylR2

Statistics are for the 20 lowestenergy structures

${ }^{a}$ Restrains distance differences and implies twofold symmetry (Nilges 1993)

b Determined with MOLMOL (Koradi et al. 1996)

c Evaluated with XPLOR-NIH (Schwieters et al. 2003)

\begin{tabular}{|c|c|c|c|c|}
\hline & a & $\mathrm{b}$ & $\mathrm{c}$ & $\mathrm{d}$ \\
\hline \multicolumn{5}{|c|}{ Intermolecular distance restraints (for each monomer) } \\
\hline Symmetry $^{\mathrm{a}}$ & - & - & - & - \\
\hline PRE & 103 & 103 & 103 & - \\
\hline NOE & - & - & 13 & 13 \\
\hline HN-RDCs & - & 57 & 57 & 57 \\
\hline \multicolumn{5}{|l|}{ After rigid body minimization } \\
\hline \multicolumn{5}{|l|}{ Rmsd from X-ray structure $(3-63)^{\mathrm{b}}$} \\
\hline Backbone atoms $(\AA)$ & 3.01 & 1.45 & 1.37 & 2.03 \\
\hline All heavy atoms $(\AA)$ & 3.61 & 2.31 & 2.27 & 2.86 \\
\hline \multicolumn{5}{|l|}{ After refinement in explicit solvent } \\
\hline \multicolumn{5}{|l|}{ Rmsd from X-ray structure $(3-63)^{b}$} \\
\hline Backbone atoms $(\AA)$ & 2.69 & 1.15 & 1.11 & 2.17 \\
\hline All heavy atoms $(\AA)$ & 3.42 & 2.08 & 2.04 & 2.96 \\
\hline Intermolecular energy ${ }^{\mathrm{c}}(\mathrm{kcal} / \mathrm{mol})$ & -1673.9 & -1609.6 & -1284.3 & -1437.8 \\
\hline
\end{tabular}

(residues 43-52) that contributes strongly to the dimer interface and the loop connecting helix $\alpha 3$ and $\alpha 4$ involved in DNA binding (Fig. 3). Within this loop the flexible residue $\mathrm{S} 42$ is found. Conformational flexibility in this region is likely to be important for DNA binding. In addition, crystal packing might have influenced the X-ray structure of CylR2.

Although broadening of signals due to a covalently attached spin label might be measured to high accuracy, the encoded distance information is less precise mainly due to the flexibility of the paramagnetic side chain. Efforts are being made to rigidify the spin label (Leonov et al. 2005) (or lanthanide binding tags attached to any of the termini of the protein (Wohnert et al. 2003)), but averaging of distance information remains a problem. To take into account the mobility of the tag, Clore and coworkers used a multiple-structure representation of the paramagnetic group in simulated annealing calculations (Iwahara et al. 2004). Here we chose a different strategy as the structure of the monomeric subunit of CylR2 could be determined using NOEs, RDCs and torsion angles. We measured PRE broadening in the ${ }^{15} \mathrm{~N}$-mut $(*) /{ }^{15} \mathrm{~N}$-mut(*) homodimer sample for residues that did not show any intermolecular PRE effects in the 1:1-mixed ${ }^{15} \mathrm{~N}-w t /$ mut $(*)$ sample. The intramolecular PRE broadening observed for these residues was used to determine the position of MTSL within the monomeric subunit of CylR2. Note that this is an average position of MTSL, which is in agreement with the observed intramolecular PRE broadening. For high-affinity complexes averaging of intra and intermolecular PRE broadening is very similar, and the average position of MTSL was kept fixed during rigid-body docking. In addition, unspecific binding of MTSL to the protein can be probed when experimental intramolecular PRE distances are compared with values calculated from the NOE-based structure of the monomeric subunit.

Cysteine mutations were introduced into loop regions on the basis of the 3D structure of the monomeric subunit of CylR2. Accordingly, ${ }^{15} \mathrm{~N}-\mathrm{HSQC}$ spectra of the 1:1-mixed ${ }^{15} \mathrm{~N}-w t / m u t(*)$ sample showed two peaks for residues primarily close to the site of mutation and new assignment using triple-resonance spectra was not required (Fig. 1c). On the other hand, when $\mathrm{Co}^{2+}$ was introduced as a paramagnetic probe sub-stoichiometrically into a homodimer, the symmetry was broken, signals from three species (the $\mathrm{Co}^{2+}$-free, the diamagnetic and two non-equivalent monomeric species) were present and resonances in the paramagnetic molecules were shifted due to pseudo contact shifts. Thus, signal overlap was strongly increased even at $900 \mathrm{MHz}$ and a 3D HNCO was required to assign the paramagnetically shifted resonances (Gaponenko et al. 2002).

\section{NMR-based ranking of homodimer structures obtained from ab initio docking}

Preparation of single-cysteine mutants is time consuming, mutations can alter the protein structure and they may not be possible due to the presence of essential cysteine residues in the $w t$ protein. Thus, it is desirable to prepare only one single-cysteine mutant of the protein of interest or completely avoid the need for intermolecular distance information. Due to the reduced amount of experimental information, however, convergence to a near native structure using conventional structure calculation protocols such as Xplor-NIH is difficult. In case of CylR2, the Xplor-NIH docking did not converge to the correct solution when only 
a

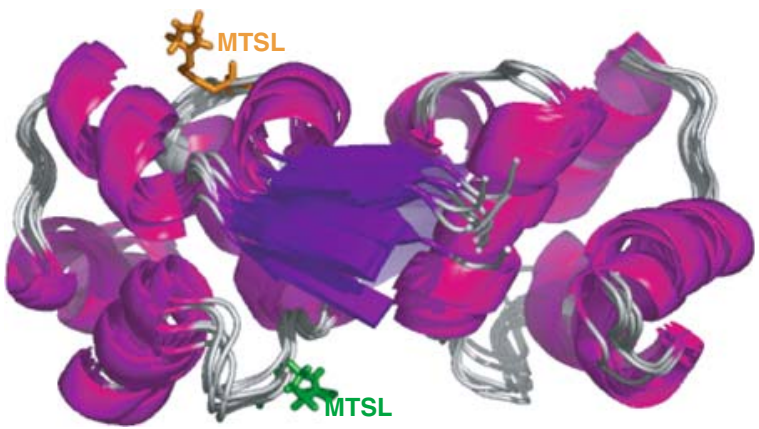

b
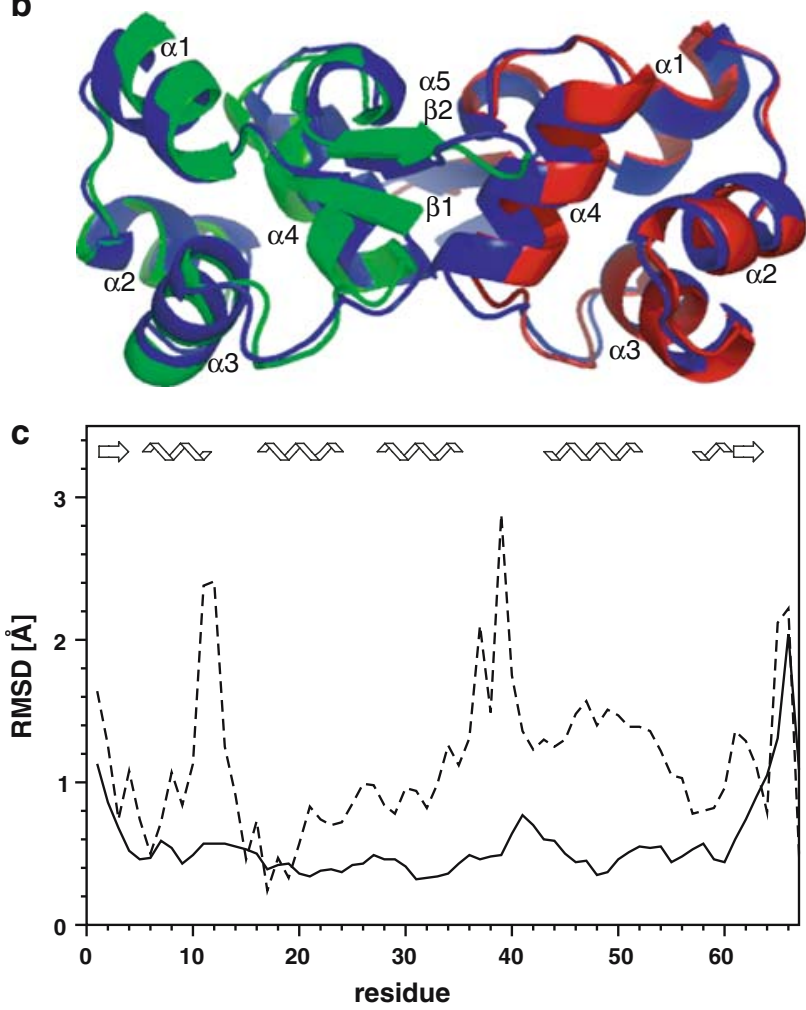

Fig. 3 High-resolution structure of the CylR2 homodimer in solution. (a) Superposition of the 10 NMR structures with lowest energy. Helices are shown in magenta and $\beta$-strands in violet. The calculated average position of MTSL attached to position N40C (green) or position T55C (orange) is indicated for the left subunit. (b) Mean structure of the NMR ensemble (blue) superimposed on the X-ray structure (red). (c) Average backbone rmsd per residue for the 15 NMR structures (solid line) and backbone rmsd per residue between the mean NMR structure and the X-ray structure (dashed line) (Rumpel et al. 2004). The rmsd values between the NMR and the $\mathrm{X}$-ray structure were calculated from the structural fit shown in (b) and are shown for both subunits of the CylR2 homodimer. Secondary structure elements are indicated

intermolecular distances for one spin-label position together with HN-RDCs and symmetry restraints were used (data not shown). Combination of a small number of intermolecular NOEs with chemical shift perturbation data (Tang and Clore 2006) or combination of intermolecular NOEs with HN-RDCs in case of CylR2 (Table 2) did, however, result in a near native structure. This suggests that the unsuccessful Xplor-NIH docking is due to the fact that all PRE distance restraints in case of a single spin label involve the same atom. In addition, intermolecular NOEs define more precisely the dimer interface due to their shortrange information content.

Good progress has been made in ab initio docking of protein complexes including homooligomeric proteins (Gray 2006). Ab initio docking programs like DOT have an optimized energy function that includes electrostatic and non-bonded interactions as well as shape complementarity. For many systems, the algorithms produce ensembles of low energy docking solutions that contain a structural model, which deviates by $2-5 \AA$ from the real structure. To improve ranking of ab initio docking models, chemical shift perturbations and RDCs were used (Dobrodumov and Gronenborn 2003; Morelli et al. 2001). For homodimeric complexes, however, the protein cannot generally be obtained in monomeric form and chemical shift perturbations at the dimer interface are not available. Here we compare intermolecular distances obtained from a 1:1mixed ${ }^{15} \mathrm{~N}$-wt/mut $(*)$ sample of a single, spin labeled CylR2 mutant with distances observed in different docking solutions produced by ab initio docking. In addition, we predict RDCs from the three-dimensional shape and charge distribution of docking solutions using PALES. Note that RDC prediction using PALES simulates the way how a protein aligns in a charged alignment medium. This is very different from the best-fit of RDCs to the structure of docking solutions that was used for ranking heterooligomeric complexes.

$\mathrm{Ab}$ initio rigid-body docking of two monomeric CylR2 molecules was performed for both the mean structure of the NMR ensemble and a monomer taken from the dimeric X-ray structure. 25 docking solutions $\left(\right.$ dock $^{\text {NMR }}$ and dock $\left.^{\mathrm{X} \text {-ray }}\right)$, as calculated by the DOT algorithm and ranked by ClusPro (Comeau and Camacho 2005; Comeau et al. 2004), were obtained in each case. The rmsd between the docking solutions and the X-ray structure of the CylR2 homodimer varied between 1.3 and $17.5 \AA$ (Fig. 4a, e). When two copies of the monomer that was extracted from the X-ray structure were docked, the docking solution that was ranked highest (rank 1) had the smallest deviation from the high-resolution structure. In addition, docking solutions with rank 2 and 3 were also very close to the high-resolution crystal structure of CylR2 (Fig. 4e). This is in agreement with previous findings that many ab initio docking algorithm are able to reassemble protein-protein complexes, when the structures of the proteins as observed in the complex are used for docking (Gray 2006). On the other hand, when the mean structure of the NMR ensemble was used, the bestranked homodimeric docking solution deviated by about $14 \AA$ from the high-resolution structure of CylR2 
Fig. 4 NMR-based ranking of structural models obtained from $a b$ initio docking for the mean monomer structure of the NMR ensemble (a-d) and a monomer of the X-ray structure of CylR2 $(\mathbf{e}-\mathbf{h})$. (a, e) Comparison of the backbone rmsd (residues 3-63) between the X-ray structure and the $a b$ initio model with the rank assigned by ClusPro; $(\mathbf{b}, \mathbf{f})$ comparison of $\sigma$, which measures the deviation between intermolecular distances derived from PREs for the spin label at position $\mathrm{T} 55 \mathrm{C}$ and distances calculated for the docking solutions, with the backbone rmsd to the X-ray structure; (c, g) PRE-based rank of docking solutions versus the rank assigned by ClusPro; (d, h) Comparison of the rank derived by prediction of molecular alignment as implemented in PALES with the ClusPro rank of docking solutions. In (c, d, $\mathbf{g}$, h) symbols are colored according to the deviation of the $\mathrm{ab}$ initio docking model from the X-ray structure of CylR2
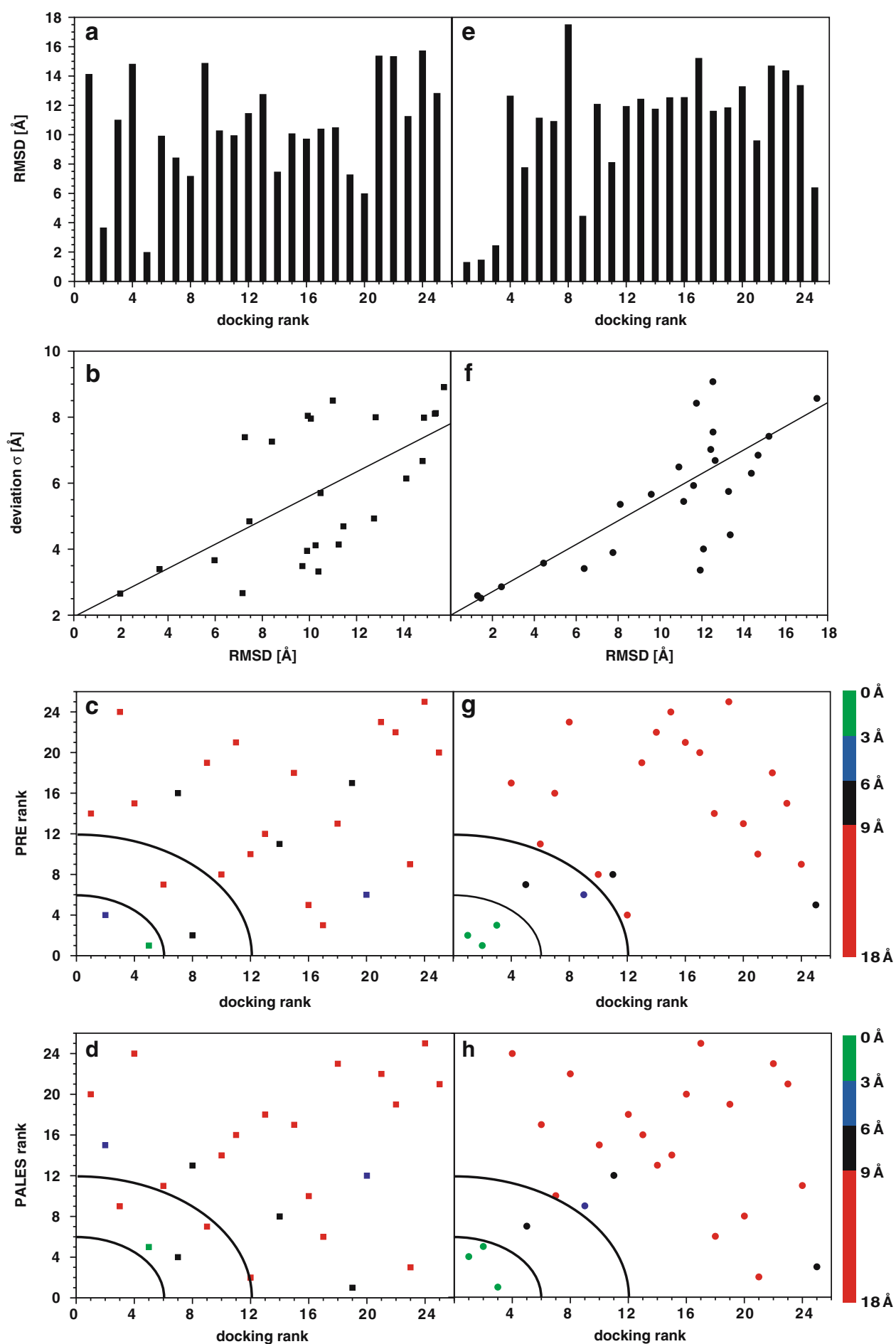

(Fig. 4a). The docking solution with the smallest deviation from the crystal structure (deviation $\sim 2 \AA$ ) was ranked only fifth. These results show that DOT/ClusPro docking is able to produce near native solutions and ranking is more reliable when the monomer is taken from the crystal structure of CylR2. At the same time, however, there is no guarantee that the solution that was ranked highest is at all similar to the real structure.
To improve ranking of homodimeric arrangements obtained from ab initio docking, we compare PRE-derived intermolecular distances with values calculated from the DOT/ClusPro solutions. For both spin label positions, the average deviation from the experimental PRE distances increases with increasing deviation of the docking model dock ${ }^{\text {NMR }}$ from the X-ray structure (Fig. 4b, f). For CylR2 ${ }_{\mathrm{T} 55 \mathrm{C}}$, the model dock ${ }^{\mathrm{NMR}}$ with the smallest deviation 
between experimental and calculated intermolecular distance restraints is the one closest to the crystal structure (deviation $\sim 2 \AA$ ) (Fig. 4b). At the same time, however, the docking model that deviates by $7.2 \AA$ from the X-ray structure fits only slightly worse to the experimental PRE distances. This is due to the estimated error of $\pm 5 \AA$ associated with the experimental PRE distances (see also Fig. 2). In addition, intermolecular distances in the docking solutions were calculated from the $\mathrm{C} \beta$ atom of the cysteine residue to which the spin label was attached. Calculation of a more accurate intermolecular distance would require positioning of the spin label using intramolecular PREs (as was done in the Xplor-NIH docking) or averaging over different side chain conformations. For CylR $2_{\mathrm{N} 40 \mathrm{C}}$, the two dock $^{\text {NMR }}$ models that fit best to experimental PRE values deviate by about 2 and $6 \AA$ from the crystal structure of CylR2 (Supplementary Fig. S2). On the other hand, the docking model that was ranked highest by ClusPro does clearly not fit to the experimental PREs observed for either CylR $2_{\mathrm{N} 40 \mathrm{C}}$ or CylR2 $2_{\mathrm{T} 55 \mathrm{C}}$ (average PRE deviations $\sigma$ of more than $6 \AA$ ). To identify the docking solution that is closest to the real structure, we propose to compare the rank assigned by the docking program ("docking rank") with the rank as obtained from the comparison with experimental PREs ("PRE rank") (Fig. 4c, g). The PRE rank was determined by sorting the docking solutions according to their average PRE deviations $\sigma$ and assigning the lowest rank to the solution that fits best to the experimental PREs. For both CylR $2_{\mathrm{N} 40 \mathrm{C}}$ and CylR2 $2_{\mathrm{T} 55 \mathrm{C}}$, only two docking models remained, for which the PRE rank and the docking rank was less than seven (Fig. 4c and Supplementary Fig. S2). Both docking models deviated by less than $4 \AA$ from the X-ray structure and the one with the smaller PRE rank was closest to the X-ray structure.

To improve ranking of docking models in the absence of a paramagnetic center, we took advantage of the possibility to predict molecular alignment tensors from the charge distribution and shape of a protein using a method implemented in the software PALES (Zweckstetter et al. 2004). Pf1 bacteriophage is strongly negatively charged and CylR2, being a DNA-binding protein, contains a patch of positive charge. Thus, the alignment orientation that was predicted by PALES for different ab initio docking models of CylR2 varied strongly. Based on the correlation between experimental RDCs and values predicted by PALES we rank the docking models and compare this PALES-based rank with the rank assigned by the ab initio docking program (Fig. 4d, h). When using the NMR monomer, only a single structure belonged to the best seven structures according to PALES-based and ab initio ranking (Fig. 4d). This docking model is closest to the $\mathrm{X}$-ray structure with a deviation of about $2 \AA$. The four docking models that were assigned a better rank according to ClusPro are not in agreement with RDCs predicted by PALES (correlation coefficients below 0.7). There is also one docking model that was assigned a docking rank of seven and a PRE rank of four, but which differs by $8.4 \AA$ from the X-ray structure of CylR2. However, when the linear average of the PRE and docking rank is calculated this docking model would obtain an average rank 2 , whereas the best docking model is ranked 1. Ranking of models, which were obtained by docking a monomer of the X-ray structure, was more reliable using either ClusPro or PALES resulting in a very reliable identification of three near native structures (Fig. 4h). The correlation between experimental RDCs and values predicted from the high-resolution NMR and X-ray structure were 0.84 and 0.80 , respectively.

In case of homodimeric coiled-coil proteins, PALES had to distinguish only between the parallel and the antiparallel arrangement (Zweckstetter et al. 2005). Moreover, due to the asymmetric distribution of charges along the chain of coiled-coil proteins the two arrangements are characterized by very different distributions of the surface charges enabling a clear distinction by PALES. In the more general case of homodimers comprised by monomers with a globular structure, many different arrangements are possible that potentially do not differ strongly in the distribution of surface charges. In addition, PALES is based on a strongly simplified electrostatic model, which might further affect the accuracy of the prediction of molecular alignment. Nevertheless, the combination of the rank assigned by PALES based on prediction of molecular alignment and the rank assigned by ClusPro based on electrostatic and desolvation energies provides a reliable approach for identification of near native docking models. The reliability of PALES ranking is further improved if only docking solutions are taken into account for which the correlation coefficient between experimental and predicted RDCs is above 0.7. Comparison of Fig. $4 \mathrm{c}$ with $4 \mathrm{~d}$ and of $4 \mathrm{~g}$ with $4 \mathrm{~h}$ indicates that PRE-based ranking is not significantly better than ranking by PALES. Thus, it is possible to identify a near native conformation without experimental information about the dimer interface using a small number of easily accessible HN-RDCs.

\section{Concluding remarks}

Our study shows that truly high-resolution structures of homodimeric proteins can be obtained by the combined use of intermolecular long-range distances obtained from paramagnetic relaxation enhancement and orientational information encoded by RDCs. Usage of PRE broadening avoids the need for assignment of side chain resonances and overcomes difficulties of distinguishing inter and intramonomer contacts in homooligomeric proteins. This is 
particularly important for trimeric and higher homooligomeric systems and high molecular weight complexes in general, in which side chain resonance assignment becomes increasingly difficult and essential deuteration limits the availability of NOE data. For high molecular weight homodimers, the structure determination of the monomeric unit by conventional NOE-based methods will also be more difficult and intramolecular PREs obtained on the same single-cysteine mutant proteins will be useful. Larger proteins have broad lines already in the diamagnetic state and an increase in line width due to a paramagnetic center may be too small to be measured accurately especially for longer distances. In this case, longitudinal amide proton relaxation enhancements $R_{1}$ might be more practical.

It appears that for homooligomeric systems, in which the symmetry can be restrained, the quality of structures obtained by ab initio docking is at least comparable to that obtained from two sets of PRE-based intermolecular distances. Only when RDCs are also included high-resolution structures can be obtained from the experimental restraints. On the other hand, attaching the spin label to only one site in the protein is generally not sufficient to obtain a correct homooligomeric structure in conventional restrained molecular dynamics simulations, even when RDCs were measured. Additional experimental restraints such intermolecular NOEs or pseudo contact shifts are then required.

Structural models obtained from ab initio rigid body docking can be reliably ranked using intermolecular distances derived from a single spin labeled position. Importantly, however, near native structures can be identified without chemical shift perturbation data and without intermolecular distances from a small set of backbone RDCs.

Acknowledgments We thank Michael S. Gilmore for providing the coding sequence of CylR2, Karin Giller for excellent technical help and Christian Griesinger for discussions. This work was supported by the Max Planck society and by a DFG Heisenberg grant (ZW 71/2-1 and 3-1) to M.Z.

\section{References}

Battiste JL, Wagner G (2000) Utilization of site-directed spin labeling and high-resolution heteronuclear nuclear magnetic resonance for global fold determination of large proteins with limited nuclear overhauser effect data. Biochemistry 39:5355-5365

Bertini I, Couture MM, Donaire A, Eltis LD, Felli IC, Lucinat C, Piccioli M, Rosato A (1996a) The solution structure refinement of the paramagnetic reduced high-potential iron-sulfur protein I from Ectothiorhodospira halophila by using stable isotope labeling and nuclear relaxation. Eur J Biochem 241:440-452

Bertini I, Felli IC, Lucinat C, Rosato A (1996b) A complete relaxation matrix refinement of the solution structure of a paramagnetic metalloprotein: reduced HiPIP I from Ectothiorhodospira halophila. Proteins 24:158-164
Bertini I, Donaire A, Lucinat C, Rosato A (1997) Paramagnetic relaxation as a tool for solution structure determination: Clostridium pasteurianum ferredoxin as an example. Proteins 29:348-358

Bewley CA, Clore GM (2000) Determination of the relative orientation of the two halves of the domain-swapped dimer of cyanovirin- $\mathrm{N}$ in solution using dipolar couplings and rigid body minimization. J Am Chem Soc 122:6009-6016

Cavanagh J (1996) Protein NMR spectroscopy: principles and practice. Academic Press, San Diego

Clore GM (2000) Accurate and rapid docking of protein-protein complexes on the basis of intermolecular nuclear Overhauser enhancement data and dipolar couplings by rigid body minimization. Proc Natl Acad Sci U S A 97:9021-9025

Comeau SR, Camacho CJ (2005) Predicting oligomeric assemblies: $\mathrm{N}$-mers a primer. J Struct Biol 150:233-244

Comeau SR, Gatchell DW, Vajda S, Camacho CJ (2004) ClusPro: an automated docking and discrimination method for the prediction of protein complexes. Bioinformatics 20:45-50

Cornilescu G, Delaglio F, Bax A (1999) Protein backbone angle restraints from searching a database for chemical shift and sequence homology. J Biomol NMR 13:289-302

Delaglio F, Grzesiek S, Vuister GW, Zhu G, Pfeifer J, Bax A (1995) NMRPipe: a multidimensional spectral processing system based on UNIX pipes. J Biomol NMR 6:277-293

Diaz-Moreno I, Diaz-Quintana A, De la Rosa MA, Ubbink M (2005) Structure of the complex between plastocyanin and cytochrome $\mathrm{f}$ from the cyanobacterium Nostoc sp. PCC 7119 as determined by paramagnetic NMR. The balance between electrostatic and hydrophobic interactions within the transient complex determines the relative orientation of the two proteins. J Biol Chem 280:18908-18915

Dobrodumov A, Gronenborn AM (2003) Filtering and selection of structural models: combining docking and NMR. Proteins 53:18-32

Dominguez C, Boelens R, Bonvin AM (2003) HADDOCK: a proteinprotein docking approach based on biochemical or biophysical information. J Am Chem Soc 125:1731-1737

Dominguez C, Bonvin AM, Winkler GS, van Schaik FM, Timmers HT, Boelens R (2004) Structural model of the UbcH5B/CNOT4 complex revealed by combining NMR, mutagenesis, and docking approaches. Structure 12:633-644

Donaldson LW, Skrynnikov NR, Choy WY, Muhandiram DR, Sarkar B, Forman-Kay JD, Kay LE (2001) Structural characterization of proteins with an attached ATCUN motif by paramagnetic relaxation enhancement NMR spectroscopy. J Am Chem Soc 123:9843-9847

Dyson HJ, Wright PE (1998) Equilibrium NMR studies of unfolded and partially folded proteins. Nat Struct Biol 5(Suppl):499-503

Englander SW, Mayne L, Bai Y, Sosnick TR (1997) Hydrogen exchange: the modern legacy of Linderstrom-Lang. Protein Sci 6:1101-1109

Feeney J, Birdsall B, Bradbury AF, Biekofsky RR, Bayley PM (2001) Calmodulin tagging provides a general method of using lanthanide induced magnetic field orientation to observe residual dipolar couplings in proteins in solution. J Biomol NMR 21:41-48

Gaponenko V, Howarth JW, Columbus L, Gasmi-Seabrook G, Yuan J, Hubbell WL, Rosevear PR (2000) Protein global fold determination using site-directed spin and isotope labeling. Protein Sci 9:302-309

Gaponenko V, Altieri AS, Li J, Byrd RA (2002) Breaking symmetry in the structure determination of (large) symmetric protein dimers. J Biomol NMR 24:143-148

Gilmore MS, Segarra RA, Booth MC (1990) An Hlyb-type function is required for expression of the Enterococcus faecalis hemolysin bacteriocin. Infect Immun 58:3914-3923 
Goodsell DS, Olson AJ (2000) Structural symmetry and protein function. Annu Rev Biophys Biomol Struct 29:105-153

Gray JJ (2006) High-resolution protein-protein docking. Curr Opin Struct Biol 16:183-193

Guntert P (2004) Automated NMR structure calculation with CYANA. Methods Mol Biol 278:353-378

Haas W, Shepard BD, Gilmore MS (2002) Two-component regulator of Enterococcus faecalis cytolysin responds to quorum-sensing autoinduction. Nature 415:84-87

Ishima R, Torchia DA (2003) Extending the range of amide proton relaxation dispersion experiments in proteins using a constanttime relaxation-compensated CPMG approach. J Biomol NMR 25:243-248

Ishima R, Torchia DA (2005) Error estimation and global fitting in transverse-relaxation dispersion experiments to determine chemical-exchange parameters. J Biomol NMR 32:41-54

Iwahara J, Clore GM (2006) Detecting transient intermediates in macromolecular binding by paramagnetic NMR. Nature 440:1227-1230

Iwahara J, Schwieters CD, Clore GM (2004) Characterization of nonspecific protein-DNA interactions by $1 \mathrm{H}$ paramagnetic relaxation enhancement. J Am Chem Soc 126:12800-12808

Kay LE, Muhandiram DR, Farrow NA, Aubin Y, Forman-Kay JD (1996) Correlation between dynamics and high affinity binding in an SH2 domain interaction. Biochemistry 35:361-368

Koradi R, Billeter M, Wuthrich K (1996) MOLMOL: a program for display and analysis of macromolecular structures. J Mol Graph 14:51-55, 29-32

Kosen PA (1989) Spin labeling of proteins. Methods Enzymol 177:86-121

Laskowski RA, Rullmannn JA, MacArthur MW, Kaptein R, Thornton JM (1996) AQUA and PROCHECK-NMR: programs for checking the quality of protein structures solved by NMR. J Biomol NMR 8:477-486

Leonov A, Voigt B, Rodriguez-Castaneda F, Sakhaii P, Griesinger C (2005) Convenient synthesis of multifunctional EDTA-based chiral metal chelates substituted with an S-mesylcysteine. Chemistry 11:3342-3348

Liepinsh E, Baryshev M, Sharipo A, Ingelman-Sundberg M, Otting G, Mkrtchian S (2001) Thioredoxin fold as homodimerization module in the putative chaperone ERp29: NMR structures of the domains and experimental model of the $51 \mathrm{kDa}$ dimer. Structure 9:457-471

Mandell JG, Roberts VA, Pique ME, Kotlovyi V, Mitchell JC, Nelson E, Tsigelny I, Ten Eyck LF (2001) Protein docking using continuum electrostatics and geometric fit. Protein Eng 14: $105-113$

Matsuda T, Ikegami T, Nakajima N, Yamazaki T, Nakamura H (2004) Model building of a protein-protein complexed structure using saturation transfer and residual dipolar coupling without paired intermolecular NOE. J Biomol NMR 29:325-338

Morelli XJ, Palma PN, Guerlesquin F, Rigby AC (2001) A novel approach for assessing macromolecular complexes combining soft-docking calculations with NMR data. Protein Sci 10: 2131-2137

Murray BE (1990) The life and times of the Enterococcus. Clin Microbiol Rev 3:46-65
Nilges M (1993) A calculation strategy for the structure determination of symmetric dimers by 1H NMR. Proteins 17:297-309

Petros AM, Mueller L, Kopple KD (1990) NMR identification of protein surfaces using paramagnetic probes. Biochemistry 29:10041-10048

Razeto A, Giller K, Haas W, Gilmore MS, Zweckstetter M, Becker S (2004) Expression, purification, crystallization and preliminary crystallographic studies of the Enterococcus faecalis cytolysin repressor CylR2. Acta Crystallogr D Biol Crystallogr 60:746-748

Rumpel S, Razeto A, Pillar CM, Vijayan V, Taylor A, Giller K, Gilmore MS, Becker S, Zweckstetter M (2004) Structure and DNA-binding properties of the cytolysin regulator CylR2 from Enterococcus faecalis. EMBO J 23:3632-3642

Russell RB, Alber F, Aloy P, Davis FP, Korkin D, Pichaud M, Topf M, Sali A (2004) A structural perspective on protein-protein interactions. Curr Opin Struct Biol 14:313-324

Sakakura M, Noba S, Luchette PA, Shimada I, Prosser RS (2005) An NMR method for the determination of protein-binding interfaces using dioxygen-induced spin-lattice relaxation enhancement. J Am Chem Soc 127:5826-5832

Schwieters CD, Kuszewski JJ, Tjandra N, Clore GM (2003) The Xplor-NIH NMR molecular structure determination package. J Magn Reson 160:65-73

Takahashi H, Nakanishi T, Kami K, Arata Y, Shimada I (2000) A novel NMR method for determining the interfaces of large protein-protein complexes. Nat Struct Biol 7:220-223

Tang C, Clore GM (2006) A simple and reliable approach to docking protein-protein complexes from very sparse NOE-derived intermolecular distance restraints. J Biomol NMR 36:37-44

Tjandra N, Bax A (1997) Direct measurement of distances and angles in biomolecules by NMR in a dilute liquid crystalline medium. Science 278:1111-1114

van Dijk M, van Dijk AD, Hsu V, Boelens R, Bonvin AM (2006) Information-driven protein-DNA docking using HADDOCK: it is a matter of flexibility. Nucleic Acids Res 34:3317-3325

Vaynberg J, Qin J (2006) Weak protein-protein interactions as probed by NMR spectroscopy. Trends Biotechnol 24:22-27

Volkov AN, Ferrari D, Worrall JA, Bonvin AM, Ubbink M (2005) The orientations of cytochrome $\mathrm{c}$ in the highly dynamic complex with cytochrome b5 visualized by NMR and docking using HADDOCK. Protein Sci 14:799-811

Wohnert J, Franz KJ, Nitz M, Imperiali B, Schwalbe H (2003) Protein alignment by a coexpressed lanthanide-binding tag for the measurement of residual dipolar couplings. J Am Chem Soc 125:13338-13339

Zweckstetter M, Bax A (2000) Prediction of sterically induced alignment in a dilute liquid crystalline phase: aid to protein structure determination by NMR. J Am Chem Soc 122:3791-3792

Zweckstetter M, Hummer G, Bax A (2004) Prediction of chargeinduced molecular alignment of biomolecules dissolved in dilute liquid-crystalline phases. Biophys J 86:3444-3460

Zweckstetter M, Schnell JR, Chou JJ (2005) Determination of the packing mode of the coiled-coil domain of cGMP-dependent protein kinase Ialpha in solution using charge-predicted dipolar couplings. J Am Chem Soc 127:11918-11919 